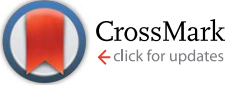

Cite this: RSC Adv., 2017, 7, 16608

Received 4th February 2017

Accepted 28th February 2017

DOI: 10.1039/c7ra01441d

rsc.li/rsc-advances

\section{Protein-derived carbon nanodots with an ethylenediamine-modulated structure as sensitive fluorescent probes for $\mathrm{Cu}^{2+}$ detection}

\begin{abstract}
Liman Sai, ${ }^{a}$ Jun Chen, ${ }^{b}$ Quanhong Chang, ${ }^{a}$ Wangzhou Shi, ${ }^{a}$ Qi Chen ${ }^{a}$ and Lei Huang ${ }^{\star a}$
The increasing use of fluorescent carbon nanodots (CNDs) demonstrates their advantages for sensing applications; these include superior photostability, absence of toxicity, and rapid analytical capability. However, CNDs usually have multiple types of functional groups covering their surfaces, which decrease their sensitivity and selectivity. In this study, the required structure of $\beta$-lactoglobulin (LG)-derived carbon nanodots was achieved by adding ethylenediamine (EDA) to the synthesis process. Due to the consumption of protein carboxyl groups during the preparation process, a homogeneous coverage of amino groups on the surfaces of the CNDs was achieved, resulting in high sensitivity of the CNDs to copper ions. Furthermore, owing to effective passivation of trap states and a high content of $\mathrm{N}$ doping, the as-prepared CNDs showed a high quantum yield and an excitation-independent emission property. We believe that this type of preparation method is useful for the design of protein-derived CNDs, which may have promising applications for detecting various metal ions in a biological environment.
\end{abstract}

\section{Introduction}

Copper ion $\left(\mathrm{Cu}^{2+}\right)$ is a transition metal ion that is essential to human health and that performs an important role in a range of fundamental physiological processes in living organisms. ${ }^{1}$ However, excessive uptake of $\mathrm{Cu}^{2+}$ can lead to diseases such as Alzheimer's, Wilson's, or Menkes' diseases. ${ }^{2}$ According to the U.S Environmental Protection agency (EPA), the average concentration of $\mathrm{Cu}^{2+}$ in blood is 15.7-23.6 $\mu \mathrm{M}$ (ref. 3) in a normal human population, and the upper limit of $\mathrm{Cu}^{2+}$ in drinking water is $20 \mu \mathrm{M} .^{4}$ Therefore, there is a great demand for simple, rapid, sensitive, and selective detection of $\mathrm{Cu}^{2+}$. Traditional techniques for detecting metal ions include atomic absorption spectroscopy, plasma mass spectrometry, and electrochemical sensors. These methods suffer from defects such as complex sample preparation, requiring well-skilled professional operators, and expensive equipment. Recently, carbonbased nanoparticles as a simple, cheap, and sensitive fluorescent probe for detecting metal ions have attracted great attention, due to their unique properties, such as superior photostability, non-toxicity, good biocompatibility, simple synthesis, and rapid analytical capability. ${ }^{5-11}$

The sensing principle of CNDs in the detection of metal ions is based on fluorescence quenching of CNDs, which derives

ajoint Lab with Wuhu Token for Graphene Electrical Materials and Application, Department of Physics, Shanghai Normal University, Guilin Road 100, Shanghai 200234, China. E-mail: leihuang@shnu.edu.cn; Fax: +86-021-64328968

${ }^{b}$ Department of Orthopedic Sport Medicine, Huashan Hospital, Fudan University, Shanghai, 200040, China from a charge transfer or energy transfer process through conjugation of ions on the surfaces of the CNDs. Selectivity is achieved through the specificity of particular ions or functional groups on the surfaces of CNDs. Thus, to achieve high sensitivity and selectivity of a CND, the its surface should be covered with a large amount of a single type of functional group. However, CNDs prepared by various methods so far usually have multiple types of functional groups on their surfaces, ${ }^{12-14}$ which limits their sensitivity and specificity to metal ions. Some types of CNDs even showed a response to different types of ions from those anticipated, ${ }^{15,16}$ and the explanation for this remained unclear.

Moreover, CNDs usually have a relatively low quantum yield compared to traditional semiconductor quantum dots ${ }^{17-19}$ and show an excitation-dependent emission, ${ }^{20-22}$ which means that the fluorescence peak position shifts with a change of excitation wavelength. A series of specific energies between LUMO and HOMO related to surface traps were believed to cause this phenomenon. Generally, the strategies for passivation of trap states were based on post-treatment of surface groups. ${ }^{23,24}$ However, these methods suffered from drawbacks including complex reaction steps, limited control of the synthesis process, and functionalized groups occupying the reactive groups on the surfaces of CNDs.

Commonly-used bottom-up methods to synthesize CNDs always include dehydration of amino and carboxylate groups followed by aromatization and carbonization. ${ }^{25-27}$ Proteins, natural bio-macromolecules possessing an abundance of functional groups, have been used as effective carbon sources to construct CNDs. ${ }^{18,22,28-30}$ In these previous works, similar 
methods were applied to form CNDs, using bovine serum albumin (BSA) as the main carbon source for providing functional groups, and denaturing agents such as urea, acetic acid, or ethanol to transform the proteins into unfolded peptide chains. However, these CNDs mostly showed excitationdependent emissions with relatively low quantum yields. ${ }^{18,22,28}$ Furthermore, various surface groups such as $-\mathrm{OH},-\mathrm{COOH}$, and $-\mathrm{NH}_{2}$ were present together on the surface of the CNDs, as indicated by FTIR and XPS data. ${ }^{18,29}$ This was probably due to limited control of the synthesis process in these studies. In any event, only a small number of protein-derived CNDs were applied to the sensing of metal ions, ${ }^{22,29}$ and the mechanism was not discussed.

Herein, we developed a simple and green synthetic approach for preparing water-soluble, fluorescent carbon nanodots derived from $\beta$-lactoglobulin (LG) by hydrothermal treatment. Compared to BSA, LG is smaller, less hydrophobic, and highly resistant to proteolytic degradation. Thus, LG has been used as a capping agent for synthesis of $\mathrm{Ag}_{2} \mathrm{~S}^{31}$ and $\mathrm{PbS}^{32}$ quantum dots. Additionally, ethylenediamine (EDA) was selected, on account of the reactive $-\mathrm{NH}_{2}$ groups at both ends of its carbon chain. EDA is an organic compound which has been widely used for preparation of carbon-based nanoparticles. ${ }^{33,34}$ A formation mechanism was proposed and confirmed by UV-vis, PL, FTIR, and XPS data. Due to the consumption of protein carboxyl groups during the synthesis process, a uniform coverage of $-\mathrm{NH}_{2}$ groups on the surface of CNDs was achieved, and the CNDs showed a sensitive response to $\mathrm{Cu}^{2+}$. Moreover, with the reduction of trap states and a high content of $\mathrm{N}$ doping, the asprepared CNDs showed a high quantum yield, and the emission from LOMO to HOMO dominated the fluorescence spectra, resulting in an excitation-independent property.

\section{Experimental}

\section{Materials}

$\beta$-Lactoglobulin (LG, molecular weight $\sim 18 \mathrm{kDa}$ ) was purchased from Sigma (USA). Ethylenediamine (EDA) was purchased from Shanghai Chemical Reagents Company (China). Milli-Q water was used as the solvent for all solutions. All reagents were used without further purification.

\section{Preparation of carbon nanodots}

Carbon nanodots were prepared in aqueous solution by means of a one-step hydrothermal method. Typically, $100 \mathrm{mg}$ of $\beta$ lactoglobulin was dissolved in $10 \mathrm{~mL}$ of deionized water. Then, a further $10 \mathrm{~mL}$ of water, which contained a specific amount of ethylenediamine $(0-750 \mu \mathrm{L})$, was added dropwise to the above LG solution under stirring. The LG protein can be denatured and transformed into linear peptide polymers under alkaline conditions. ${ }^{22}$ In the absence of EDA, the pH value of the LG solution was adjusted to 11 with $20 \mu \mathrm{L}$ of concentrated $\mathrm{NaOH}$. In the presence of EDA (from 15-750 $\mu \mathrm{L}$ ), the $\mathrm{pH}$ value already exceeded 11 so no further adjustment was applied. The mixed solution then underwent hydrothermal synthesis at $200{ }^{\circ} \mathrm{C}$ for $7 \mathrm{~h}$. The as-prepared CNDs were cooled naturally to room temperature and concentrated by rotary evaporation. Purification was achieved by dialysis $(0.5 \mathrm{KD})$ for about 2 days, and the obtained CNDs were stored at $4{ }^{\circ} \mathrm{C}$.

\section{Selective detection of metal ions}

To investigate the effect of metal ions on the fluorescence property of CNDs, different types of metal salts were used as follows: $\mathrm{Zn}\left(\mathrm{NO}_{3}\right)_{2}, \mathrm{FeCl}_{2}, \mathrm{FeCl}_{3}, \mathrm{Cu}\left(\mathrm{NO}_{3}\right)_{2}, \mathrm{Hg}\left(\mathrm{NO}_{3}\right)_{2}, \mathrm{~Pb}\left(\mathrm{NO}_{3}\right)_{2}$, $\mathrm{Mg}\left(\mathrm{NO}_{3}\right)_{2}, \mathrm{CdCl}_{2}$, and $\mathrm{CaCl}_{2}$. Typically, $1 \mathrm{~mL}$ aqueous solutions of $450 \mu \mathrm{M}$ metal salts were freshly prepared and mixed with 2 $\mathrm{mL}$ of diluted CNDs solution. The absorbance values of CNDs in the mixed solution were set to 0.1 . The fluorescence measurement was performed after equilibrating for $20 \mathrm{~min}$.

\section{Structural and optical characterizations}

Transmission electron microscopy (TEM) and high-resolution TEM (HRTEM) images were recorded from a JEOL-2011 electron microscope (JEOL, Tokyo, Japan) with an accelerating voltage of $200 \mathrm{kV}$. Samples for TEM and HRTEM were prepared by dropping CNDs solution onto a carbon-coated copper grid. Powder X-ray diffraction (XRD) spectra were measured on a Philips X'Pert diffractometer using $\mathrm{Cu} \mathrm{K} \alpha$ radiation. Fourier transform infrared (FTIR) spectra were recorded on a ThermoNicolet transform infrared spectrometer (Nicolet/Nexus 670). $\mathrm{X}$-ray photoelectron spectroscopy (XPS) analyses were carried out using an ESCALAB 250 spectrometer equipped with a monochromatic X-ray source with $\mathrm{Al} \mathrm{K} \alpha$ excitation (1486.6 eV). CNDs powder for XRD, FTIR, and XPS samples was obtained by freeze drying. UV-vis absorption spectra were recorded using a Lambda 950 UV-vis spectrophotometer (Perkin Elmer, USA). Photoluminescence (PL) spectra were obtained using a Shimadzu RF-6310PC spectrofluorometer (Shimadzu Corporation, Columbia, MD, USA). Time-resolved fluorescence decay curves were obtained using a Horiba Jobin Yvon single-photon counting system with a $375 \mathrm{~nm}$ diode and a $1 \mathrm{MHz}$ repetition rate and 1.3 ns pulse width.

\section{Calculation of fluorescence quantum yield}

Quinine sulfate dissolved in $0.1 \mathrm{M} \mathrm{H}_{2} \mathrm{SO}_{4}$ (literature quantum yield $=54 \%$ ) was selected as a reference standard. The quantum yield of the CNDs was calculated by comparing the integrated photoluminescence intensities (excited at $320 \mathrm{~nm}$ ) and the absorbance values at $320 \mathrm{~nm}$ of the CNDs with the reference quinine sulfate. Absorbance values for all the solutions were measured at the excitation wavelength. Then, a graph was plotted of the integrated fluorescence intensity against the absorbance and a trend line was produced for each curve, with its intercept at zero. Absolute values were calculated according to the following equation:

$$
\phi_{\mathrm{X}}=\phi_{\mathrm{ST}}\left(\frac{K_{\mathrm{X}}}{K_{\mathrm{ST}}}\right)\left(\frac{\eta_{\mathrm{X}}^{2}}{\eta_{\mathrm{ST}}{ }^{2}}\right)
$$

where the subscripts ST and X denote standard and test respectively, $\phi$ is the quantum yield, $K$ is the gradient from the plot of integrated fluorescence intensity $v s$. absorbance, and $\eta$ is the refractive index of the solvent. In order to minimize re- 
absorption effects, the absorbance value of the CNDs solution was set below 0.1 at the excitation wavelength of $320 \mathrm{~nm}$.

\section{Results and discussion}

\section{Structure characterization}

The structure and surface groups were confirmed through TEM, HRTEM, and FTIR analysis. TEM and HRTEM images of CNDS are shown in Fig. 1. The CNDs had spherical shape and good monodispersity (Fig. 1a-c). The average diameter of the nanoparticles was measured as about $8.42 \mathrm{~nm}$. The well-resolved lattice fringe in HRTEM images (Fig. 1d and e) confirmed the good crystalline nature of the CNDs. Fig. 2 shows the powder XRD pattern of the CNDs. The broad diffractive peak at $18.21^{\circ}$ revealed the amorphous nature of the $\mathrm{CNDs},{ }^{20}$ while the peak at $26.18^{\circ}$ (d002) was attributed to highly disordered carbon, similar to graphite lattice spacing. ${ }^{23,26,35}$

\section{Optical properties}

To investigate the optical properties of the CNDs prepared in the presence of EDA, UV-vis spectra of the CNDs prepared with different amounts of EDA are shown in Fig. 3. Without EDA, the protein can also form fluorescent nanodots (named as LG-CND) in basic solution, with a weak absorption shoulder which may come from residual protein, as pure LG showed an absorption peak at about $280 \mathrm{~nm}$. However, we believe that the peaks at $280 \mathrm{~nm}$ in the curves of CNDs prepared with EDA (named as EDA-CND) originated from the $n-\pi *$ transition $^{23}$ of $\mathrm{C}=\mathrm{O}$ bonds in the carbonic core rather than in the LG protein, because with a small amount of EDA $(15 \mu \mathrm{L})$, the peak at $280 \mathrm{~nm}$ disappeared, due to complete denaturing of the protein. A new absorption band was observed in EDA-CND at $317 \mathrm{~nm}$, which can be assigned to the $\pi-\pi^{*}$ transition of surface groups. ${ }^{26}$ As supplementary data, UV-vis spectra of the CNDs before and after
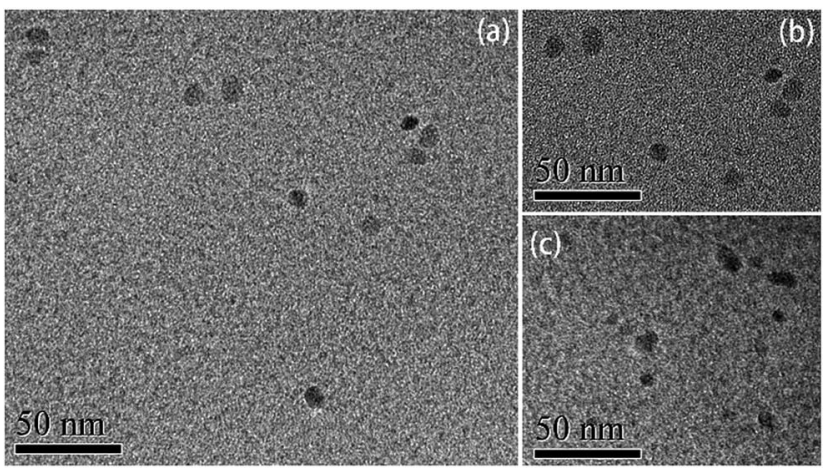

(d)

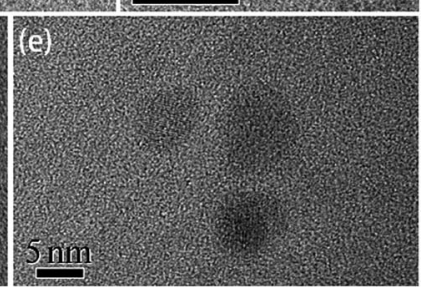

Fig. 1 TEM (a-c) and HRTEM ( $d$ and e) images of the CNDs prepared with the addition of $300 \mu \mathrm{L}$ of EDA.

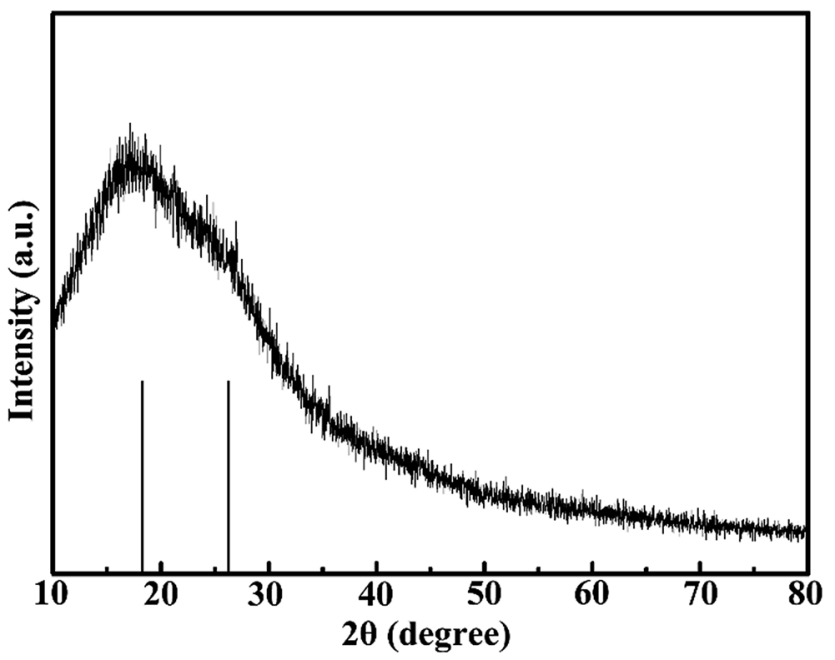

Fig. 2 XRD pattern of CNDs. The black bars at the bottom represent the peak position of the pattern.

dialysis were recorded. As impurities in solution are likely to adsorb to the surface of CNDs, the intensity of the $317 \mathrm{~nm}$ peak, which is related to surface groups, should decrease after purification, which is consistent with the data (inset in Fig. 3). With additional amounts of EDA, the ratio of the intensity of the $280 \mathrm{~nm}$ peak to that of the $317 \mathrm{~nm}$ peak gradually increased. Based on the above discussion, this indicated formation of the graphite structure and ordering of the surfaces of the CNDs.

The PL spectra of CNDs excited by various excitation wavelengths are shown in Fig. 4. In the cases of no EDA (Fig. 4a) and $15 \mu \mathrm{L}$ addition of EDA (Fig. 4b), the CNDs clearly showed excitation-dependent emission. There existed multiple peaks upon high-energy excitation, and trap emission could be found in the wavelength range of $600-800 \mathrm{~nm}$, indicating multiple transition modes of the CNDs due to various energy levels. These energy levels were probably caused by formation of

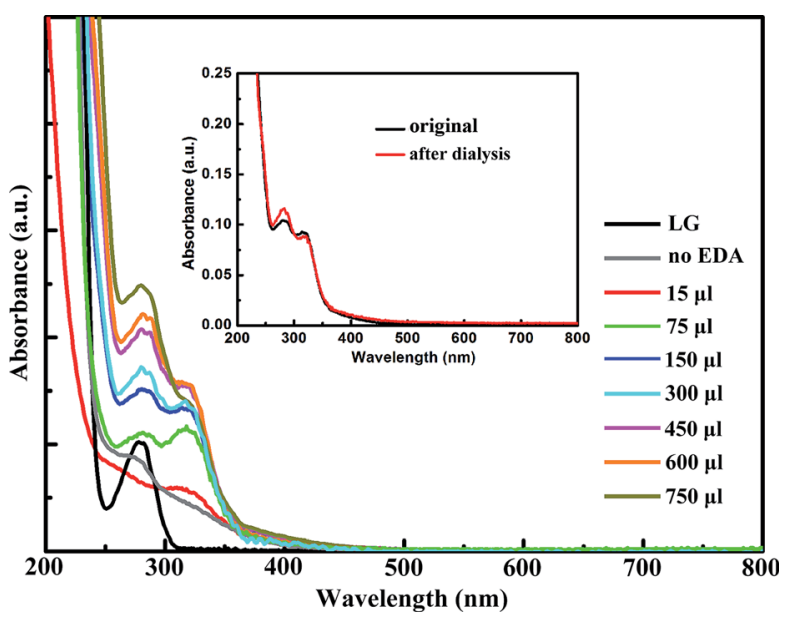

Fig. 3 UV-vis absorption spectra of CNDs synthesized with different added amounts of EDA. The insert picture shows absorption spectra of CNDs (synthesized with $300 \mu \mathrm{L}$ of added EDA) before and after $500 \mathrm{D}$ dialysis. 

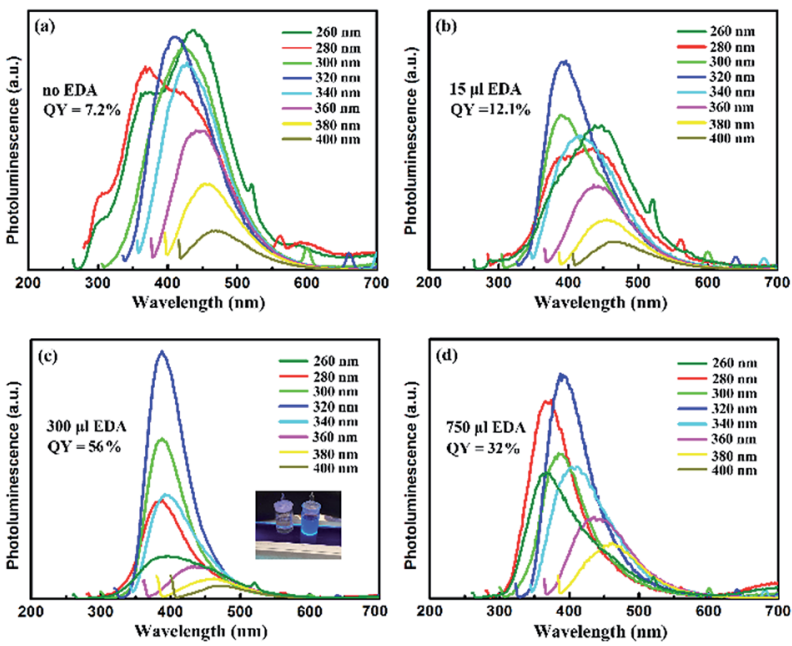

Fig. 4 PL spectra of CNDs under excitation with different wavelengths. The CNDs were synthesized with (a) no EDA, (b) $15 \mu \mathrm{L}$ EDA, (c) $300 \mu \mathrm{L} \mathrm{EDA}$ and (d) $750 \mu \mathrm{L} \mathrm{EDA}$. The absorption values of the solution were all adjusted to 0.1 . Inset in c: the photograph of a CND solution in daylight (left) and under UV light (300 nm) (right).

a disordered structure during the intermolecular dehydration process and poor passivation of the surfaces of CNDs. On the other hand, the position of the emission peaks for CNDs synthesized with $300 \mu \mathrm{L}$ addition of EDA remained almost unchanged in the excitation wavelength range of $280-340 \mathrm{~nm}$ (Fig. 4c). Further increase of excitation wavelength led to a redshift of the emission wavelength as well as a large decrease in the fluorescence intensity. Surface states are known to introduce emission levels between HOMO and LUMO, which cause the excitation-dependent behaviour of CNDs. ${ }^{36,37}$ Thus, the emission occurring with an excitation wavelength above $340 \mathrm{~nm}$ can be assigned to the trap states. The low fluorescence intensity of these peaks indicated the relatively low proportion of trap emission compared to the intrinsic emission that came from the radiative transition of $\mathrm{sp}^{2}$ carbon. With a further increase in the amount of EDA, the emission of CNDs again showed excitation dependence (Fig. 4d). This was caused by excess amino groups on the surfaces of the CNDs giving rise to non-radiative defects, which is commonly observed in the case of semiconductor quantum dots. ${ }^{38}$

To further investigate the fluorescence property of the CNDs, lifetime measurements were carried out, and the PL decay curves of LG-CND and EDA-CND are shown in Fig. 5. As discussed above, upon excitation at $375 \mathrm{~nm}$, the emission at $444 \mathrm{~nm}$ (LG-CND) and $458 \mathrm{~nm}$ (EDA-CND) originated from trap states. As shown in Fig. 5, an obvious decrease in lifetime was observed in CNDs prepared with EDA. The decay curve can be fitted into a multiexponential function with three lifetimes. Fitting parameters are listed in Table 1. The average lifetime was calculated using eqn (2):

$$
\langle\tau\rangle=\frac{\left(\alpha_{1} \tau_{1}^{2}+\alpha_{2} \tau_{2}^{2}+\alpha_{3} \tau_{3}^{2}\right)}{\alpha_{1} \tau_{1}+\alpha_{2} \tau_{2}+\alpha_{3} \tau_{3}}
$$

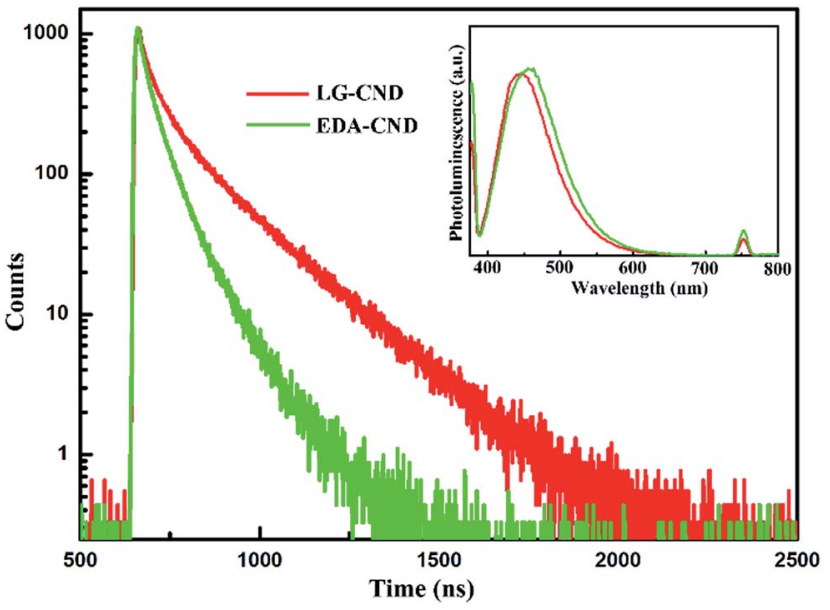

Fig. 5 Fluorescence decay curves of CNDs synthesized with (300 $\mu \mathrm{L})$ and without EDA. The lifetimes were recorded at the maxima of the emission with the excitation wavelength of $375 \mathrm{~nm}$. The insert picture indicates the PL spectra of the corresponding CNDs.

Table 1 Fitting parameters of the emission decay curves of LG-CND and EDA-

\begin{tabular}{lcccc}
\hline & $\tau_{1} / \mathrm{ns}$ & $\tau_{2} / \mathrm{ns}$ & $\tau_{3} / \mathrm{ns}$ & $\langle\tau\rangle / \mathrm{ns}$ \\
\hline LG-CND & 2.23 & 6.89 & 21.69 & 9.76 \\
EDA-CND & 1.30 & 4.46 & 10.61 & 4.68 \\
\hline
\end{tabular}

The average lifetimes of LG-CND and EDA-CND were calculated to be $9.76 \mathrm{~ns}$ and $4.68 \mathrm{~ns}$, respectively. The reduced lifetime of trap emission in EDA-CND indicated the effective passivation of trap states by EDA. Moreover, in agreement with the PL and lifetime spectra, the quantum yield of the CNDs reached the highest value of $56 \%$ with addition of $300 \mu \mathrm{L}$ of EDA. A photograph of CNDs showing strong blue emission under a UV lamp is shown in the inset of Fig. 4c. To the best of our knowledge, this is the highest quantum yield for CNDs derived from protein. ${ }^{18,22,28-30}$

\section{Characterization of surface groups}

Next, FTIR and XPS spectroscopy were used to investigate the surface functional groups of CNDs. FTIR spectra of LG protein, LG-CND, and EDA-CND are shown in Fig. 6. In all three curves, $\mathrm{O}-\mathrm{H}$ and $-\mathrm{CH}_{2}-$ groups were confirmed by peaks at 3300-3500 $\mathrm{cm}^{-1}$ and $1380 \mathrm{~cm}^{-1}$, respectively. In the curves of both kinds of CNDs, a unique peak at about $810 \mathrm{~nm}$ appeared, which can be assigned to the vibration of an epoxy group, ${ }^{26}$ indicating formation of the $\mathrm{sp}^{2}$ carbonic core. In addition, the peaks at $1638 \mathrm{~cm}^{-1}, 1578 \mathrm{~cm}^{-1}$, and $1482 \mathrm{~cm}^{-1}$ can be assigned to the amide I $\mathrm{C}=\mathrm{O},{ }^{20}$ amide $\mathrm{II} \mathrm{N}-\mathrm{H},{ }^{17,39}$ and amide III C-N stretching vibrations, ${ }^{40}$ respectively. These peaks confirmed the formation of amide bonds, resulting from a dehydration reaction between carboxyl and amino groups. Comparing the two kinds of CNDs, in the curve for EDA-CND the absorption intensity of the $\mathrm{N}-\mathrm{H}$ peak was stronger, whereas the intensity of the $\mathrm{C}=\mathrm{O}$ peak was 


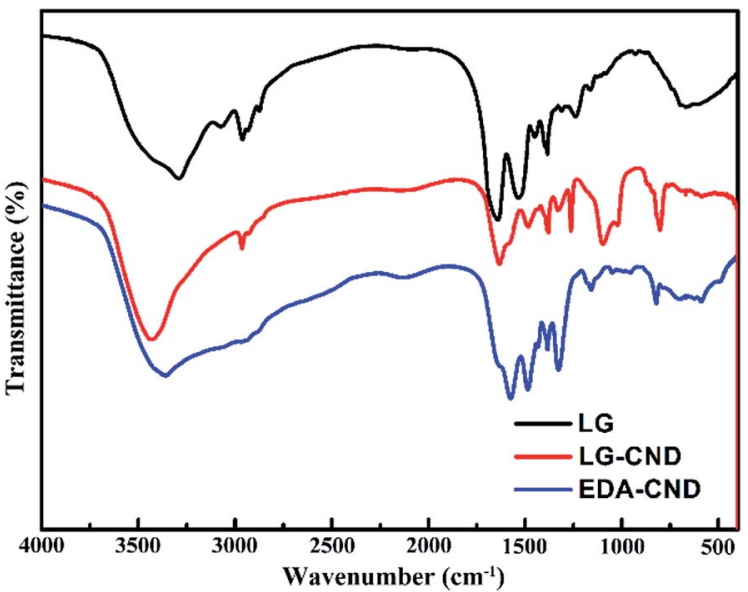

Fig. 6 FTIR spectra of LG protein and CNDs synthesized with and without EDA.

weaker, than in the curve for LG-CND. This indicated that more amino groups were present in EDA-CND.

To further investigate the surface groups of CNDs, XPS measurements of LG-CND (Fig. 7a-c) and EDA-CND (Fig. 7d-f) were carried out. In the spectra of both kinds of CNDs, the C1s spectrum revealed different types of carbon atoms: graphitic $(\mathrm{C}=\mathrm{C})$ or aliphatic $(\mathrm{C}-\mathrm{C})$; oxygenated $(\mathrm{C}-\mathrm{O})$; and nitrous $(\mathrm{C}=\mathrm{O}$ or $\mathrm{C}=\mathrm{N}) \cdot{ }^{18}$ Moreover, the N1s spectrum showed two peaks which were attributed to aromatic $\mathrm{N}$ and surface $-\mathrm{NH}_{2}$ groups, respectively. ${ }^{23}$ In the spectrum of EDA-CND, the oxygen content decreased while the carbon content increased (Table 2), and the peak intensity of $\mathrm{C}=\mathrm{C} / \mathrm{C}-\mathrm{C}$ was higher (Fig. $7 \mathrm{~b}$ and e). This
Table 2 Compositions of LG-CND and EDA-CND from XPS analysis

\begin{tabular}{lllr}
\hline & $\mathrm{C}$ & $\mathrm{O}$ & \multicolumn{1}{c}{$\mathrm{N}$} \\
\hline LG-CND & $66.29 \%$ & $24.00 \%$ & $9.71 \%$ \\
EDA-CND & $73.24 \%$ & $15.04 \%$ & $11.72 \%$
\end{tabular}

indicated a higher content of a graphite structure, due to more adequate carbonization, which is consistent with the UV-vis data in Fig. 3. Meanwhile, the nitrogen content in EDA-CND was higher (Table 2), and in the N1s spectrum (Fig. 7c and f), the ratio of the peak intensities for $-\mathrm{NH}_{2}$ and $-\mathrm{CONH}$ was larger. This provided evidence that there existed more surface $-\mathrm{NH}_{2}$ groups in EDA-CND, which would be important for $\mathrm{Cu}^{2+}$ ion detection.

\section{Sensitive detection of $\mathrm{Cu}^{2+}$}

The interaction between CNDs and $\mathrm{Cu}^{2+}$ was investigated by optical characterization. In the UV-vis spectra in Fig. 8, the CNDs had two absorption bands centered at 280 and $317 \mathrm{~nm}$. However, after adding $150 \mu \mathrm{M} \mathrm{Cu}^{2+}$, the absorption band at $280 \mathrm{~nm}$ disappeared, and another new broad band in the range from 500 to $700 \mathrm{~nm}$ (inset in Fig. 8a and b) appeared. This indicated combination of $\mathrm{Cu}^{2+}$ ions with the amino groups on the surfaces of CNDs, which was similar to other CNDs with abundant amino groups in a previous report. ${ }^{41}$ Furthermore, in the PL spectra in Fig. 8, addition of $\mathrm{Cu}^{2+}$ ions gave rise to a significant decrease in the fluorescence intensity of EDA-CND (Fig. 8b), while $\mathrm{Cu}^{2+}$ at the same concentration had little effect on the fluorescence of LG-CND (Fig. 8a). The UV-vis and PL data
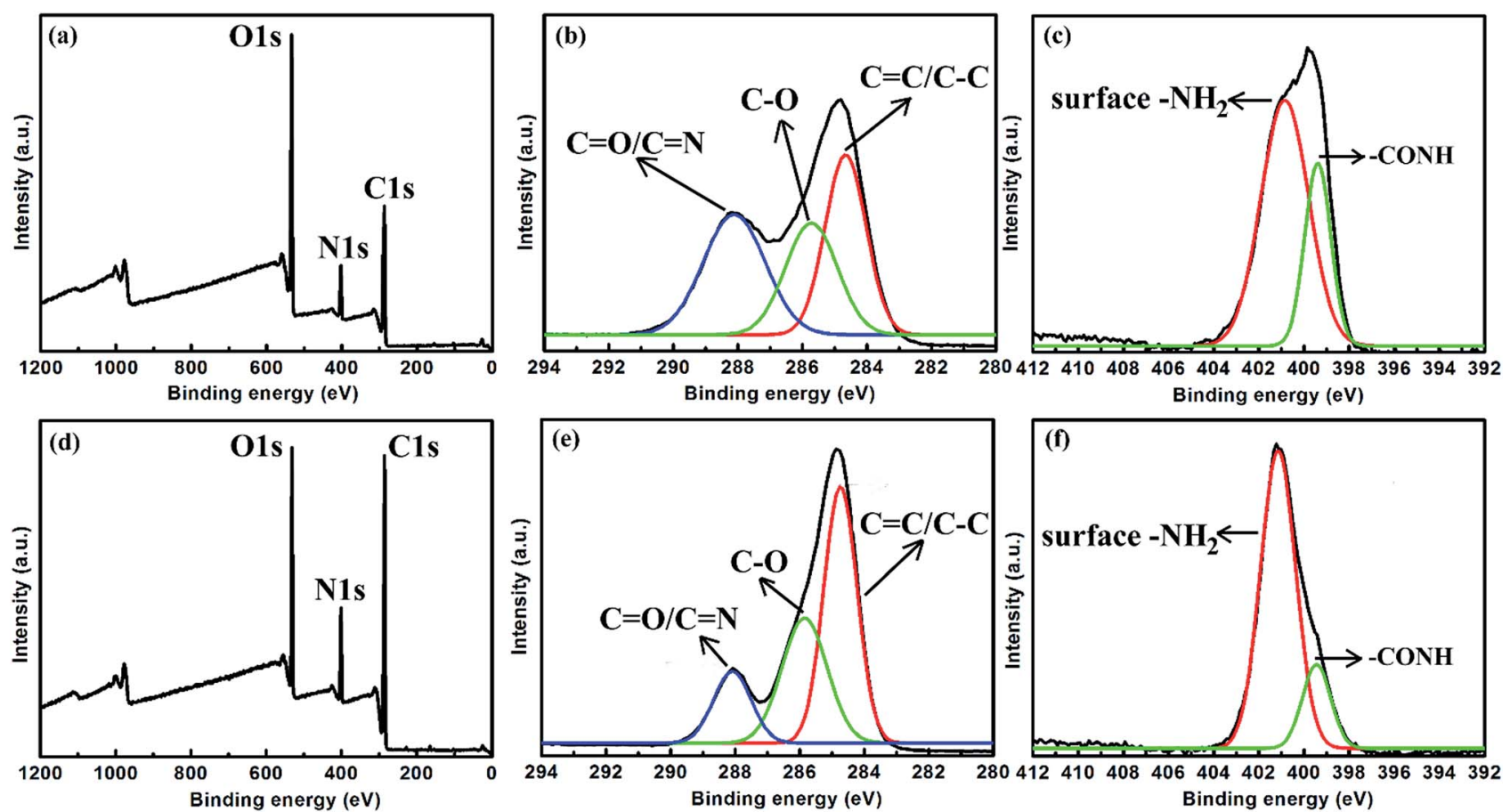

Fig. 7 XPS survey spectra of (a) LG-CND and (d) EDA-CND. The detailed (high-resolution) scans of (b) C1s and (c) N1s of LG-CND. The detailed (high-resolution) scans of (e) C1s and (f) N1s of EDA-CND. 

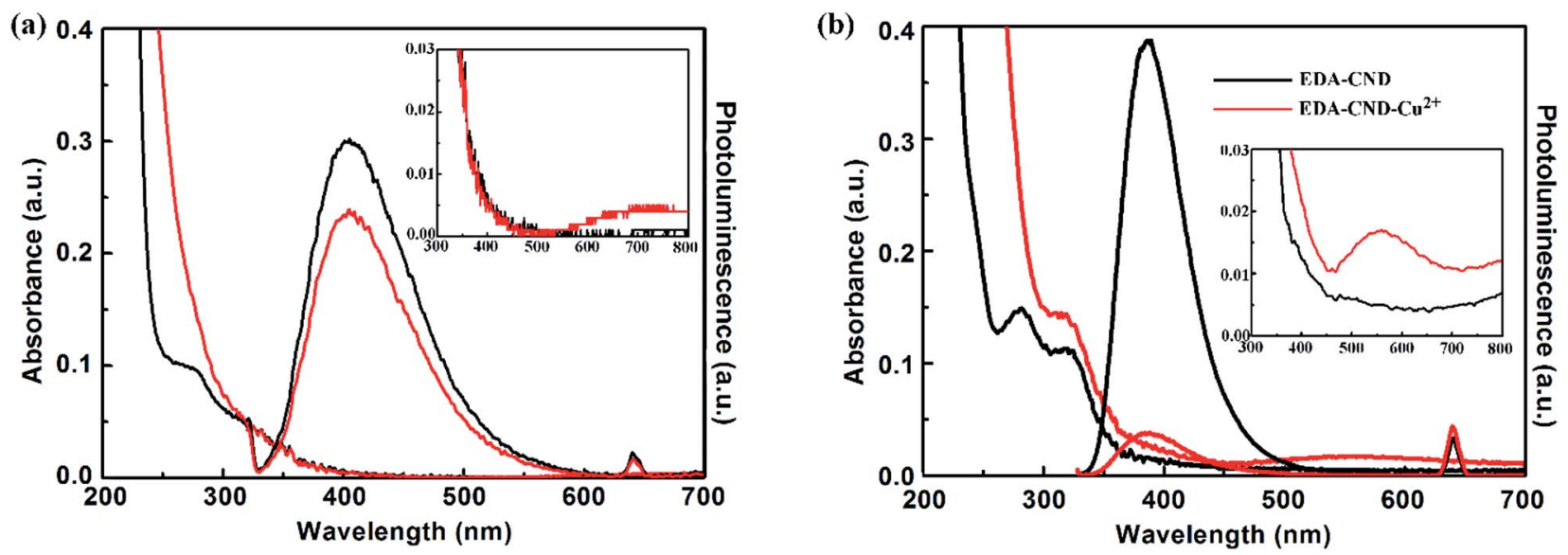

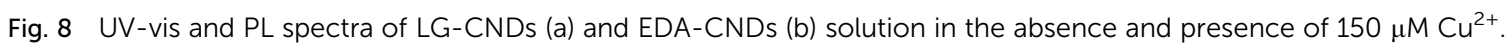

clearly indicated the strong interaction of $\mathrm{Cu}^{2+}$ with the amino groups on the surfaces of EDA-CND.

The selectivity of the EDA-CND sensing system was then evaluated. A variety of metal ions including $\mathrm{Cd}^{2+}, \mathrm{Hg}^{2+}, \mathrm{Fe}^{2+}$, $\mathrm{Fe}^{3+}, \mathrm{Pb}^{2+}, \mathrm{Ca}^{2+}, \mathrm{Mg}^{2+}$, and $\mathrm{Zn}^{2+}$ were examined. Fig. 9 shows the fluorescence intensity of CNDs solutions containing $\mathrm{Cu}^{2+}$ and other metal ions $(150 \mu \mathrm{M})$. It can be seen that other metal ions had little influence on the fluorescence of CNDs, indicating that the CNDs were highly selective for $\mathrm{Cu}^{2+}$ as compared to other metal ions.

To determine the detection limit, different concentrations of $\mathrm{Cu}^{2+}$ ranging from 10 to $60 \mu \mathrm{M}$ were mixed with CNDs, and the corresponding fluorescence spectra were measured after $20 \mathrm{~min}$. The relationship between $I / I_{0}$ and concentration of $\mathrm{Cu}^{2+}$ is shown in Fig. 10. The quenching effect can be described by the Stern-Volmer eqn (3):

$$
\frac{I_{0}}{I}=1+K_{\mathrm{s}}[\mathrm{C}]
$$

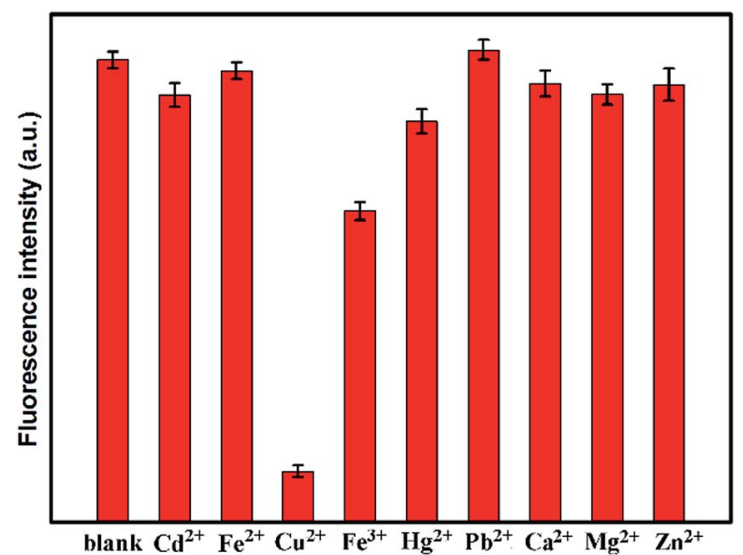

Fig. 9 Selectivity of EDA-CNDs for $\mathrm{Cu}^{2+}$ over other metal ions. The concentration of metal ions were $150 \mu \mathrm{M}$. The absorption value of all the mixed solutions were fixed at 0.1. All measurements were recorded at $387 \mathrm{~nm}$. where $K_{\mathrm{s}}$ is the Stern-Volmer quenching constant, and $I_{0}$ and $I$ are the fluorescence intensities recorded at $387 \mathrm{~nm}$ with and without $\mathrm{Cu}^{2+}$, respectively. A linear correlation existed over the range of $\mathrm{Cu}^{2+}$ concentrations from 10 to $60 \mu \mathrm{M}\left(R^{2}=0.997\right)$. The detection limit $(3 \sigma / K)$ was determined to be $116 \mathrm{nM}$.

\section{Mechanism of formation of CNDs}

Based on the data and the discussion above, we propose a formation mechanism for the CNDs (Scheme 1). In general, the LG protein was first denatured into unfolded peptide chains in basic solution (Scheme 1a). With increasing heating time, dehydration of the amino groups in the EDA and of carboxyl groups in the protein occurred (Scheme $1 \mathrm{~b}$ ), $\mathrm{C}=\mathrm{C}$ bonds were formed inside the peptide network, resulting in aromatic clusters (Scheme 1c), and nucleation of CNDs took place (Scheme $1 \mathrm{~d}) .{ }^{22}$

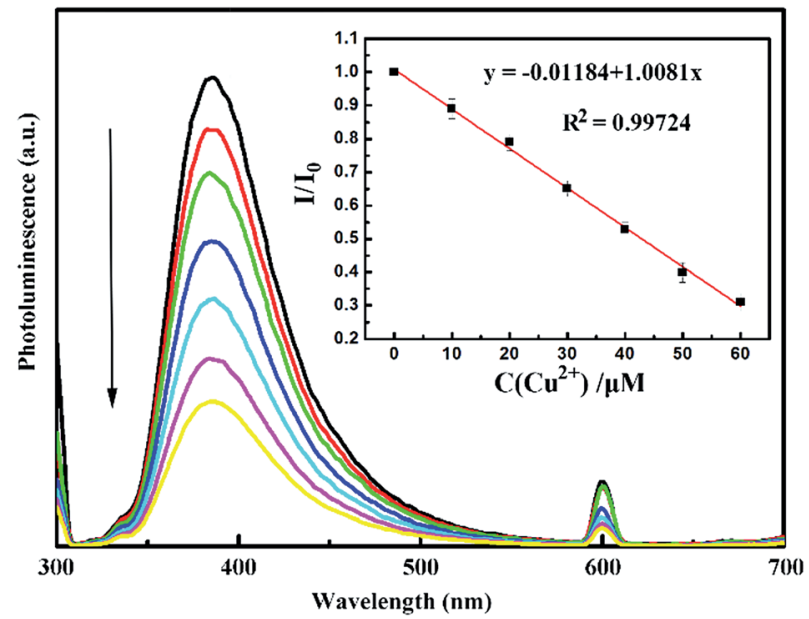

Fig. 10 Fluorescence quenching of CNDs (synthesized with $300 \mu \mathrm{L}$ addition of EDA) by various concentrations of $\mathrm{Cu}^{2+}$ (from top to bottom: 0, 10, 20, 30, 40, 50, and $60 \mu \mathrm{M})$. Inset: Stern-Volmer plot of the fluorescence quenching of CNDs solution by $\mathrm{Cu}^{2+}$ over the range of $10-60 \mu \mathrm{M}$. The absorption value of the CNDs solution was 0.1 . 


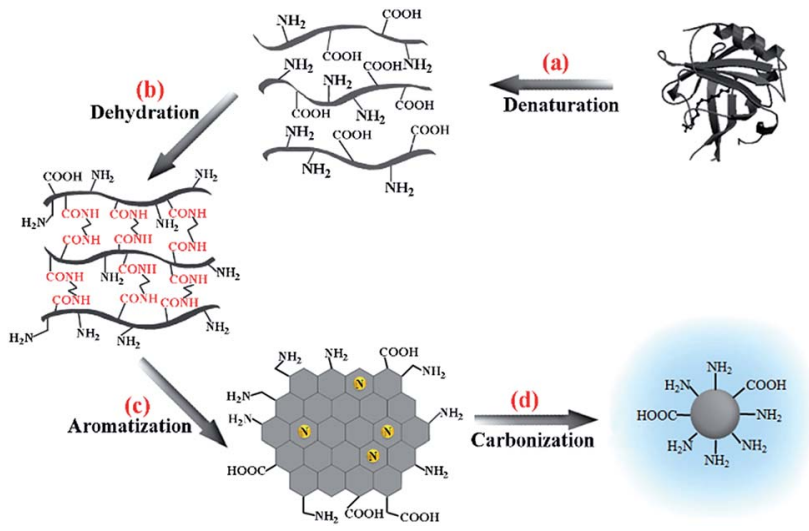

Scheme 1 A formation mechanism for CNDs derived from $\beta$-lactoglobulin in the presence of ethylenediamine.

In previous studies involving protein-derived CNDs, the synthesis process was based on intermolecular dehydration between peptide chains of the protein, while various other denaturing reagents, ${ }^{22}$ crosslinking ${ }^{18}$ reagents, or a stabilizer ${ }^{28}$ were used. In this work, EDA can act both as the denaturing agent and as a reaction precursor for synthesis of the CNDs. EDA is an organic compound with strong basicity and two reactive amino groups, and it has been proven to be an efficient precursor for preparing CNDs. Previous studies also found EDA to be optimal in comparison with various other aminocontaining molecules, when molecules containing single amino groups led to inadequate condensation polymerization. ${ }^{26}$ As shown in Scheme 1b, in the presence of EDA, amino groups at both ends of the short chain of EDA could react with carboxyl groups in the LG protein, and the peptide chains of the protein were then linked by EDA instead of through intermolecular crosslinking. Carbonization then took place and resulted in CNDs with strong fluorescence.

Our results revealed that EDA played a key role in the improvement of both optical properties and sensitivity to $\mathrm{Cu}^{2+}$ of the CNDs. The reason can be explained as follows. First, the regular structure obtained by this strategy could result in fewer defects inside the CNDs, and the degree of $\mathrm{N}$ doping was improved by incorporation of amine groups into the CNDs (confirmed by XPS data), which was believed to be responsible for high luminescence of CNDs. ${ }^{42,43}$ In addition, $-\mathrm{NH}_{2}$ groups exposed on the surface of CNDs could act as surface passivation agents which reduced the surface trap states. ${ }^{23}$ As a consequence, the EDA-CNDs showed excitation-independent emission and high quantum yield when compared to LG-EDA. The strong fluorescence and stable emission of the CND paved the way for its application in sensing.

Most importantly, the high alkalinity of the EDA molecule, which means strong protonation of $-\mathrm{NH}_{2}$ groups in aqueous solution, facilitated its interaction with $-\mathrm{COOH}$ groups in the LG protein. ${ }^{26}$ However, in previous studies, the long chain of TTDDA, ${ }^{28}$ or the weak protonation of $-\mathrm{NH}_{2}$ groups in urea, ${ }^{22}$ prevented full participation in the reaction. In previous accounts, CNDs prepared from proteins have seldom been reported to be sensitive to $\mathrm{Cu}^{2+}{ }^{18,22,28-30}$ The interference of diverse types of surface groups in CNDs prepared directly from proteins could reduce their sensitivity to $\mathrm{Cu}^{2+}$, which specifically bonds with $-\mathrm{NH}_{2}$ groups. In our work, the participation of EDA in the formation of CNDs led to consumption of the carboxyl groups in the protein, and resulted in a homogeneous coverage of $-\mathrm{NH}_{2}$ groups on the surfaces of the CNDs obtained (confirmed by FTIR and XPS data). As revealed by the data, the high sensitivity and selectivity of our CNDs to $\mathrm{Cu}^{2+}$ ions provided evidence for the proposed mechanism.

\section{Conclusions}

In summary, a hydrothermal treatment of $\beta$-lactoglobulin in the presence of ethylenediamine has been proven to be an effective strategy for producing fluorescent CNDs. During the synthesis process, EDA acted as both denaturing agent and reaction precursor. Owing to the high degree of $\mathrm{N}$ doping and surface passivation by amino groups, the as-prepared CNDs showed excitation-independent emission and high quantum yield. Moreover, the surface groups of the CNDs can be adjusted through varying the amount of EDA used. Due to the consumption of $-\mathrm{COOH}$ groups of the LG protein during synthesis of the CNDs, the surfaces of the CNDs were homogeneously covered with $-\mathrm{NH}_{2}$ groups. Finally, the CNDs obtained showed high sensitivity and good selectivity for the detection of $\mathrm{Cu}^{2+}$. On account of their superior optical characteristics and sensitivity, these CNDs are very promising for applications in sensing.

\section{Acknowledgements}

This research was supported by the Natural Science Foundation of Shanghai (No. 16ZR1424400), Shanghai Municipal Education Committee Key Laboratory of Molecular Imaging Probes and Sensors for Shanghai Universities, and the Industrial Research Fund of Wuhu Token Sciences Co., Ltd. Prof. Lei Huang appreciates the support of the Program for Professor of Special Appointment (Eastern Scholar) at Shanghai Institutions of Higher Learning.

\section{Notes and references}

1 E. L. Que, D. W. Domaille and C. J. Chang, Chem. Rev., 2008, 108, 1517-1549.

2 K. J. Barnham, C. L. Masters and A. I. Bush, Nature, 2004, 3, 205-214.

3 H. S. Jung, P. S. Kwon, J. W. Lee, J. I. Kim, C. S. Hong, J. W. Kim, S. H. Yan, J. Y. Lee, J. H. Lee, T. Joo and J. S. Kim, J. Am. Chem. Soc., 2009, 131, 2008-2012.

4 R. Uauy, M. Olivares and M. Gonzalez, Am. J. Clin. Nutr., 1998, 67, 952s-959s.

5 G. Gedda, C. Y. Lee, Y. C. Lin and H. F. Wu, Sens. Actuators, B, 2016, 224, 396-403.

6 J. Chen, Y. Li, K. Lv, W. Zhong, H. Wang, Z. Wu, P. Yi and J. Jiang, Sens. Actuators, B, 2016, 224, 298-306. 
7 Y. Li, X. Q. Liu, Q. Y. Li, J. Ge, H. Liu, S. Li, L. F. Wang, J. Wang and N. Ma, Chem. Phys. Lett., 2016, 664, 127-132.

8 W. Lu, X. Qin, S. Liu, G. Chang, Y. Zhang, Y. Luo, A. M. Asiri, A. O. Al-Youbi and X. Sun, Anal. Chem., 2012, 84, 5351-5357.

9 J. Tian, Q. Liu, A. M. Asiri, A. O. Al-Youbi and X. Sun, Anal. Chem., 2013, 85, 5595-5599.

10 L. Zhou, Y. Lin, Z. Huang, J. Ren and X. Qu, Chem. Commun., 2012, 48, 1147-1149.

11 H. Sun, N. Gao, L. Wu, J. Ren, W. Wei and X. Qu, Chem.-Eur. J., 2013, 19, 13362-13368.

12 L. L. Li, J. Ji, R. Fei, C. Z. Wang, Q. Lu, J. R. Zhang, L. P. Jiang and J. J. Zhu, Adv. Funct. Mater., 2012, 22, 2971-2979.

13 D. Wang, L. Wang, X. Y. Dong, Z. Shi and J. Jin, Carbon, 2012, 50, 2147-2154.

14 H. Tan, W. X. Liu, B. Gong, W. Zhang, H. D. Li, D. H. Yu, H. L. Wang, G. D. Li and L. A. Lucia, Langmuir, 2015, 31, 9537-9545.

15 S. W. Zhang, J. X. Li, M. Y. Zeng, J. Z. Xu, X. K. Wang and W. P. Hu, Nanoscale, 2014, 6, 4157-4162.

16 J. Yu, N. Song, Y.-K. Zhang, S.-X. Zhong, A.-J. Wang and J. Chen, Sens. Actuators, B, 2015, 214, 29-35.

17 H. Liu, Z. He, L.-P. Jiang and J.-J. Zhu, ACS Appl. Mater. Interfaces, 2015, 7, 4913-4920.

18 M. Tan, X. Li, H. Wu, B. Wang and J. Wu, Colloids Surf., B, 2015, 136, 141-149.

19 X. Hu, X. An and L. Li, Mater. Sci. Eng., C, 2016, 58, 730-736.

20 P.-C. Hsu and H.-T. Chang, Chem. Commun., 2012, 48, 39843986.

21 Y. Y. Zhang, M. Wu, Y. Q. Wang, X. W. He, W. Y. Li and X. Z. Feng, Talanta, 2013, 117, 196-202.

22 X. Liu, T. Li, Y. Hou, Q. Wu, J. Yi and G. Zhang, RSC Adv., 2016, 6, 11711-11718.

23 X. M. Li, S. L. Zhang, S. A. Kulinich, Y. L. Liu and H. B. Zeng, Sci. Rep., 2014, 4.

24 R. X. Tian, S. L. Hu, L. L. Wu, Q. Chang, J. L. Yang and J. Liu, Appl. Surf. Sci., 2014, 301, 156-160.

25 P. Zhang, W. C. Li, X. Y. Zhai, C. J. Liu, L. M. Dai and W. G. Liu, Chem. Commun., 2012, 48, 10431-10433.
26 S. Zhu, Q. Meng, L. Wang, J. Zhang, Y. Song, H. Jin, K. Zhang, H. Sun, H. Wang and B. Yang, Angew. Chem., Int. Ed., 2013, 52, 3953-3957.

27 X. Hu, L. Cheng, N. Wang, L. Sun, W. Wang and W. Liu, RSC Adv., 2014, 4, 18818-18826.

28 Z. Zhang, J. Hao, J. Zhang, B. Zhang and J. Tang, RSC Adv., 2012, 2, 8599-8601.

29 Q. X. Yang, L. Wei, X. F. Zheng and L. H. Xiao, Sci. Rep., 2015, 5.

30 V. B. Kumar, J. Sheinberger, Z. Porat, Y. Shav-Tal and A. Gedanken, J. Mater. Chem. B, 2016, 4, 2913-2920.

31 J. Chen, Y. Kong, Y. Wo, H. Fang, Y. Li, T. Zhang, Y. Dong, Y. Ge, Z. Wu, D. Zhou and S. Chen, J. Mater. Chem. B, 2016, 4, 6271-6278.

32 J. Chen, Y. F. Kong, W. Wang, H. W. Fang, Y. Wo, D. J. Zhou, Z. Y. Wu, Y. X. Li and S. Y. Chen, Chem. Commun., 2016, 52, 4025-4028.

33 S. Liu, J. Tian, L. Wang, Y. Luo, J. Zhai and X. Sun, J. Mater. Chem., 2011, 21, 11726-11729.

34 S. Liu, J. Tian, L. Wang, Y. Luo and X. Sun, RSC Adv., 2012, 2, 411-413.

35 Y. H. Yang, J. H. Cui, M. T. Zheng, C. F. Hu, S. Z. Tan, Y. Xiao, Q. Yang and Y. L. Liu, Chem. Commun., 2012, 48, 380-382.

36 J. Z. Shang, L. Ma, J. W. Li, W. Ai, T. Yu and G. G. Gurzadyan, Sci. Rep., 2012, 2.

37 L. Bao, Z.-L. Zhang, Z.-Q. Tian, L. Zhang, C. Liu, Y. Lin, B. Qi and D.-W. Pang, Adv. Mater., 2011, 23, 5801-5806.

38 L. H. Yuwen, H. T. Lu, Y. He, L. Q. Chen, M. Hu, B. Q. Bao, F. Boey, H. Zhang and L. H. Wang, J. Mater. Chem., 2010, 20, 2788-2793.

39 P. Karfa, E. Roy, S. Patra, S. Kumar, A. Tarafdar, R. Madhuri and P. K. Sharma, RSC Adv., 2015, 5, 58141-58153.

40 J. Jiang, Y. He, S. Li and H. Cui, Chem. Commun., 2012, 48, 9634-9636.

41 Y. Dong, R. Wang, G. Li, C. Chen, Y. Chi and G. Chen, Anal. Chem., 2012, 84, 6220-6224.

42 J. Zhou, X. Zhou, R. Li, X. Sun, Z. Ding, J. Cutler and T.-K. Sham, Chem. Phys. Lett., 2009, 474, 320-324.

43 X. Huang, L. Yang, S. Hao, B. Zheng, L. Yan, F. Qu, A. M. Asiri and X. Sun, Inorg. Chem. Front., 2017. 